\title{
On the directional entropy of $\mathbb{Z}^{2}$-actions generated by cellular automata
}

\author{
by \\ M. Courbage (Paris) and B. Kamiński (Toruń)
}

\begin{abstract}
We show that for any cellular automaton $(\mathrm{CA}) \mathbb{Z}^{2}$-action $\Phi$ on the space of all doubly infinite sequences with values in a finite set $A$, determined by an automaton rule $F=F_{[l, r]}, l, r \in \mathbb{Z}, l \leq r$, and any $\Phi$-invariant Borel probability measure, the directional entropy $h_{\vec{v}}(\Phi), \vec{v}=(x, y) \in \mathbb{R}^{2}$, is bounded above by $\max \left(\left|z_{l}\right|,\left|z_{r}\right|\right) \log \# A$ if $z_{l} z_{r} \geq 0$ and by $\left|z_{r}-z_{l}\right|$ in the opposite case, where $z_{l}=x+l y, z_{r}=x+r y$.

We also show that in the class of permutative CA-actions the bounds are attained if the measure considered is uniform Bernoulli.
\end{abstract}

Introduction. The concept of the directional entropy of a $\mathbb{Z}^{2}$-action has been introduced by Milnor [5]. For basic definitions and properties of the topological and metric directional entropy we refer the reader also to the paper [2] of Boyle and Lind.

In this paper we consider the metric directional entropy of CA-actions of $\mathbb{Z}^{2}$, i.e. $\mathbb{Z}^{2}$-actions generated by the natural extension of an automaton map (determined by an automaton rule) and the shift acting on the space of all doubly infinite sequences taking values in a finite state space.

It has been shown by Park [7] that for any CA-action the directional entropy with respect to any invariant Borel probability measure is continuous as a function of the direction vector.

Shereshevsky has shown in [9] that if the automaton map is bipermutative then the associated CA-action is strongly mixing and it determines in every direction a Bernoulli transformation with respect to the uniform Bernoulli measure.

In general the calculation of the directional entropy is not easy.

In this paper we first estimate from above the directional entropy of any $\mathrm{CA}$-action with respect to an invariant Borel probability measure.

2000 Mathematics Subject Classification: Primary 28D20; Secondary 37A15, 37A35.

Key words and phrases: cellular automata, directional entropy.

Research of B. Kamiński supported by KBN grant 2 P03A 00214. 
Next we consider permutative CA-actions, i.e. actions determined by left or right permutative or bipermutative automaton rules. It is well known that for these actions the uniform Bernoulli measure is invariant.

We prove that if the automaton rule $F_{[l, r]}$ is right permutative then $h_{\vec{v}}(\Phi)=\left|z_{r}\right| \log \# A$ for every $\vec{v}=(x, y)$ with $0 \leq z_{l} \leq z_{r}$ or $z_{r} \leq z_{l} \leq 0$, where $z_{r}=x+r y$ and $z_{l}=x+l y$. A similar result is valid for left permutative automaton rules. If $F_{[l, r]}$ is bipermutative with $z_{l} \cdot z_{r} \leq 0$ then $h_{\vec{v}}(\Phi)=$ $\left|z_{r}-z_{l}\right| \log \# A$.

In [6] Milnor gave a formula for the directional entropy in the case of bipermutative automaton rules by applying geometrical ideas. In our considerations we use elementary properties of entropy.

Result. Let $(X, \mathcal{B}, \mu)$ be a Lebesgue probability space and let $\mathcal{Z}$ be the set of all countable measurable partitions of $X$ with finite entropy equipped with the Rokhlin metric

$$
\varrho(P, Q)=H(P \mid Q)+H(Q \mid P)
$$

where $H(P \mid Q)$ stands for the conditional entropy of $P$ with respect to $Q$ for $P, Q \in \mathcal{Z}$.

Let $\xi, \eta \in \mathcal{Z}$. We write $\xi \preccurlyeq \eta$ if any atom of $\eta$ is contained in some atom of $\xi$. Let $\Phi$ be a $\mathbb{Z}^{2}$-action on $(X, \mathcal{B}, \mu)$. For a set $A \subset \mathbb{R}^{2}$ and $P \in \mathcal{Z}$ we put

$$
P(A)=\bigvee_{g \in A \cap \mathbb{Z}^{2}} \Phi^{g} P .
$$

Let $\vec{v}=(x, y)$ be a fixed vector of $\mathbb{R}^{2}$ and let $\Gamma$ denote the family of all bounded subsets of $\mathbb{R}^{2}$. Let $(T, S)$ be an ordered pair of commuting automorphisms of $X$ which generate $\Phi$, i.e.

$$
\Phi^{g}=T^{m} S^{n}, \quad g=(m, n) \in \mathbb{Z}^{2} .
$$

For a partition $P \in \mathcal{Z}$ we put

$$
h_{\vec{v}}((T, S), P)=\sup _{B \in \Gamma} \limsup _{t \rightarrow \infty} \frac{1}{t} H(P(B+[0, t) \vec{v})) .
$$

It is known (cf. [7]) that in fact

$$
h_{\vec{v}}((T, S), P)=\sup _{B \in \Gamma} \lim _{t \rightarrow \infty} \frac{1}{t} H(P(B+[0, t) \vec{v})) .
$$

If we take another pair $\left(T_{1}, S_{1}\right)$ of commuting generators of $\Phi$ such that

$$
T=T_{1}^{a} S_{1}^{b}, \quad S=T_{1}^{c} S_{1}^{d}
$$

then it is easy to see that

$$
h_{\vec{v}}((T, S), P)=h_{A(\vec{v})}\left(\left(T_{1}, S_{1}\right), P\right)
$$

where $P \in \mathcal{Z}$ and $A=\left(\begin{array}{ll}a & c \\ b & d\end{array}\right)$. 
We shall use the notation

$$
h_{\vec{v}}(\Phi, P)=h_{\vec{v}}\left(\left(T_{0}, S_{0}\right), P\right)
$$

where $T_{0}=\Phi^{(1,0)}$ and $S_{0}=\Phi^{(0,1)}$. The quantity $h_{\vec{v}}(\Phi, P)$ is called the directional mean entropy of $\Phi$ with respect to $P$ in direction $\vec{v}$.

It is not hard to show that

$$
h_{\vec{v}}(\Phi, P)=\lim _{m \rightarrow \infty} \lim _{t \rightarrow \infty} \frac{1}{t} H(P(R(\vec{v}, m, t)))
$$

where

$$
\begin{aligned}
& R(\vec{v}, m, t) \\
& = \begin{cases}\left.(i, j) \in \mathbb{Z}^{2} ; 0 \leq j \leq[t y],-m+j x / y<i \leq m+j x / y\right\} & \text { if } y \neq 0 \\
\left\{(i, j) \in \mathbb{Z}^{2} ;-m<j \leq m, 0 \leq i \leq[t x]\right. & \text { if } y=0 .\end{cases}
\end{aligned}
$$

The quantity

$$
h_{\vec{v}}(\Phi)=\sup _{P \in \mathcal{Z}} h_{\vec{v}}(\Phi, P)
$$

is called the directional entropy of $\Phi$ in direction $\vec{v}$.

It easily follows from the definition that

(1) For every $\alpha \in \mathbb{R}$,

$$
h_{\alpha \vec{v}}(\Phi)=|\alpha| h_{\vec{v}}(\Phi)
$$

(2) If $\vec{v}=(p, q) \in \mathbb{Z}^{2}$ then

$$
h_{\vec{v}}(\Phi)=h\left(\Phi^{(p, q)}\right) .
$$

Let $X$ be the space of all two-sided sequences indexed by $\mathbb{Z}$ with values in a finite set $A=\{0,1, \ldots, a-1\}$ and let $\mathcal{B}$ denote the product $\sigma$-algebra of $X$. We denote by $\tau$ the shift transformation on $X$, i.e.

$$
(\tau x)_{i}=x_{i+1}, \quad i \in \mathbb{Z} .
$$

Let $m$ be a fixed positive integer. We denote by $A^{m}$ the $m$-fold cartesian product $A \times \ldots \times A$. Any mapping $F: A^{m} \rightarrow A$ is said to be an automaton rule. An automaton rule $F$ is said to be right permutative (cf. [9]) if for any $\left(\bar{x}_{1}, \ldots, \bar{x}_{m-1}\right) \in A^{m-1}$ the mapping $x_{m} \mapsto F\left(\bar{x}_{1}, \ldots, \bar{x}_{m-1}, x_{m}\right)$ is a permutation of $A$.

A left permutative mapping is defined similarly. We say that $F$ is bipermutative if it is right and left permutative.

Let now $l, r \in \mathbb{Z}, l \leq r$, be given and let $F: A^{r-l+1} \rightarrow A$ be a fixed automaton rule. The transformation $T: X \rightarrow X$ defined by

$$
(T x)_{i}=F\left(x_{i+l}, \ldots, x_{i+r}\right)
$$

is said to be the automaton map defined by $F$. 
Let $\mu$ be a probability measure on $\mathcal{B}$ invariant with respect to $\tau$ and $T$. If $F$ is left or right permutative then by Theorem 6.6 of [4] and Theorem 2.1 of $[3]$ the uniform Bernoulli measure has the above invariance property. Let $(\widehat{X}, \widehat{\mathcal{B}}, \widehat{\mu}, \widehat{T})$ denote the natural extension of the dynamical system $(X, \mathcal{B}, \mu, T)($ cf. $[8])$.

Recall that $\widehat{T}$ is defined as follows:

$$
\widehat{T} \widehat{x}=\left(T x^{(0)}, x^{(0)}, \ldots\right), \quad \widehat{x}=\left(x^{(0)}, x^{(1)}, \ldots\right),
$$

where $T x^{(i)}=x^{(i-1)}, i \geq 1$. We put

$$
\widehat{\tau} \widehat{x}=\left(\tau x^{(0)}, \tau x^{(1)}, \ldots\right) .
$$

Obviously $\widehat{\tau} \widehat{T}=\widehat{T} \widehat{\tau}$. The $\mathbb{Z}^{2}$-action $\Phi$ generated by $\widehat{\tau}$ and $\widehat{T}$,

$$
\Phi^{(p, q)}=\widehat{\tau}^{p} \widehat{T}^{q},
$$

is said to be a $C A$-action. For these actions Park has shown in [7] that the mapping $\vec{v} \mapsto h_{\vec{v}}(\Phi)$ is continuous. For a positive integer $m$ and $E \in \mathcal{B}$ we put

$$
E^{(m)}=\left\{\widehat{x} \in \widehat{X} ; x^{(m)} \in E\right\} .
$$

It is clear that

$$
\widehat{T}^{-1} E^{(m)}=E^{(m-1)}, \quad m \geq 1 .
$$

If $\eta=\left\{E_{1}, \ldots, E_{t}\right\}$ is a measurable partition of $X$ then we denote by $\eta^{(m)}$ the measurable partition of $\widehat{X}$ defined by

$$
\eta^{(m)}=\left\{E_{1}^{(m)}, \ldots, E_{t}^{(m)}\right\} .
$$

Let $\xi$ be the zero-time partition of $X$ :

$$
\xi=\left\{C_{0}(0), \ldots, C_{0}(a-1)\right\} \quad \text { where } \quad C_{0}(i)=\left\{x \in X ; x_{0}=i\right\}, \quad i \in A .
$$

For $i, j \in \mathbb{Z}, i \leq j$, we put

$$
\xi(i, j)=\bigvee_{u=i}^{j} \tau^{-u} \xi
$$

and

$$
\xi_{m}(-m, m)=\xi(-m, m)^{(0)} \vee \ldots \vee \xi(-m, m)^{(m)}, \quad m \geq 0 .
$$

It is clear that

$$
\xi_{m}(-m, m)=\bigvee_{s=0}^{m} \widehat{T}^{-s} \xi(-m, m)^{(m)}, \quad m \geq 0,
$$

and $\left(\xi_{m}(-m, m)\right)$ is a refining sequence of partitions tending to the partition of $\widehat{X}$ into points.

We need the following two lemmas proved in [9]. Let $T: X \rightarrow X$ be the automaton map defined by an automaton rule $F_{[l, r]}$. 
Lemma 1. For any $i, j \in \mathbb{Z}, i \leq j$, we have

$$
T^{-1} \xi(i, j) \preccurlyeq \xi(i+l, j+r) .
$$

Lemma 2. If $F$ is right (resp. left) permutative, $\mu$ is the uniform Bernoulli measure and

$$
\left.\left(m-m^{\prime}\right) r>j_{1}-i_{2} \quad\left(\text { resp. }\left(m-m^{\prime}\right) l<i_{1}-j_{2}\right)\right),
$$

then the partitions $\xi\left(i_{1}, j_{1}\right)^{(m)}$ and $\xi\left(i_{2}, j_{2}\right)^{\left(m^{\prime}\right)}$ are independent.

Let $\vec{v}=(x, y) \in \mathbb{R}^{2}, z_{l}=x+l y, z_{r}=x+r y$.

Proposition. For any $C A$-action $\Phi$ and any $\Phi$-invariant Borel probability measure we have

$$
h_{\vec{v}}(\Phi) \leq \begin{cases}\max \left(\left|z_{l}\right|,\left|z_{r}\right|\right) \log a & \text { if } z_{l} \cdot z_{r} \geq 0 \\ \left|z_{r}-z_{l}\right| \log a & \text { if } z_{l} \cdot z_{r} \leq 0\end{cases}
$$

Proof. First we show the desired estimates for $\vec{v} \in \mathbb{Z}^{2}$. Let $\vec{v}=(p, q)$. We consider the following cases:

$$
\mathrm{A}: 0 \leq z_{l} \leq z_{r}, \quad \mathrm{~B}: z_{l} \leq z_{r} \leq 0, \quad \mathrm{C}: z_{l} \leq 0 \leq z_{r} .
$$

If we obtain the estimates in cases $\mathrm{A}$ and $\mathrm{B}$, then by (1) we obtain them also in the cases $z_{r} \leq z_{l} \leq 0$ and $0 \leq z_{r} \leq z_{l}$, respectively, and hence in the case $z_{l} \cdot z_{r} \geq 0$. Using the same argument we see that in case $\mathrm{C}$ it is enough to obtain the desired inequality for $z_{l} \cdot z_{r} \leq 0$. Let $i, n$ be arbitrary positive integers. We have

$$
\begin{aligned}
\bigvee_{k=0}^{n} \widehat{\tau}^{-p k} \widehat{T}^{-q k} \xi_{i}(-i, i) & =\bigvee_{k=0}^{n} \bigvee_{s=0}^{i} \widehat{\tau}^{-p k} \widehat{T}^{-(s+q k)} \xi(-i, i)^{(i)} \\
& =\bigvee_{k=0}^{n} \bigvee_{s=0}^{i} \widehat{T}^{-(s+q k)} \xi(-i+p k, i+p k)^{(i)}
\end{aligned}
$$

CASE A. The inequality $z_{l} \leq z_{r}$ is of course equivalent to $q \geq 0$. By Lemma 1 we have

$$
\begin{aligned}
\bigvee_{k=0}^{n} \widehat{\tau}^{-p k} \widehat{T}^{-q k} \xi_{i}(-i, i) & \preccurlyeq \bigvee_{k=0}^{n} \bigvee_{s=0}^{i} \xi(-i+p k+l(s+q k), i+p k+r(s+q k))^{(i)} \\
& =\bigvee_{k=0}^{n} \bigvee_{s=0}^{i} \xi\left(-i+l s+z_{l} k, i+r s+z_{r} k\right)^{(i)} \\
& \preccurlyeq \bigvee_{k=0}^{n} \xi\left(-i(|l|+1)+z_{l} k, i(|r|+1)+z_{r} k\right)^{(i)}
\end{aligned}
$$


Since $z_{l} \geq 0, z_{r} \geq 0$ we have

$$
\bigvee_{k=0}^{n} \widehat{\tau}^{-p k} \widehat{T}^{-q k} \xi_{i}(-i, i) \preccurlyeq \xi\left(-i(|l|+1), i(|r|+1)+z_{r} n\right)^{(i)} .
$$

Therefore we get the following estimate for the entropy:

$$
\begin{aligned}
H_{\widehat{\mu}}\left(\bigvee_{k=0}^{n} \widehat{\tau}^{-p k} \widehat{T}^{-q k} \xi_{i}(-i, i)\right) & \leq H_{\widehat{\mu}}\left(\xi\left(-i(|l|+1), i(|r|+1)+z_{r} n\right)^{(i)}\right) \\
& =H_{\mu}\left(\xi\left(-i(|l|+1), i(|r|+1)+z_{r} n\right)\right) \\
& \leq\left(i(|r|+|l|+2)+z_{r} n+1\right) \log a .
\end{aligned}
$$

Hence the mean entropy satisfies the inequality

$$
h_{\widehat{\mu}}\left(\xi_{i}(-i, i), \widehat{\tau}^{p} \widehat{T}^{q}\right) \leq z_{r} \log a
$$

for any $i \geq 0$. Since the sequence $\left(\xi_{i}(-i, i)\right)$ is refining and tends to the partition into points as $i \rightarrow \infty$ the well known property of the entropy gives

$$
h_{\vec{v}}(\Phi)=h\left(\widehat{\tau}^{p} \widehat{T}^{q}\right) \leq z_{r} \log a .
$$

CASE B. Since in this case we also have $q \geq 0$ we may apply the inequality obtained in case A:

$$
\bigvee_{k=0}^{n} \widehat{\tau}^{-p k} \widehat{T}^{-q k} \xi_{i}(-i, i) \preccurlyeq \bigvee_{k=0}^{n} \xi\left(-i(|l|+1)+z_{l} k, i(|r|+1)+z_{r} k\right)^{(i)}
$$

But now $z_{l} \leq 0, z_{r} \leq 0$ and so we get

$$
\bigvee_{k=0}^{n} \widehat{\tau}^{-p k} \widehat{T}^{-q k} \xi_{i}(-i, i) \preccurlyeq \xi\left(-i(|l|+1)+z_{l} n, i(|r|+1)\right)^{(i)}, \quad i, n \geq 0 .
$$

Hence, by the same reasoning as in the previous case we get

$$
h_{\vec{v}}(\Phi) \leq-z_{l} \log a=\left|z_{l}\right| \log a .
$$

CASe C. Also in this case $q \geq 0$ and we rewrite the inequality from case B:

$$
\bigvee_{k=0}^{n} \widehat{\tau}^{-p k} \widehat{T}^{-q k} \xi_{i}(-i, i) \preccurlyeq \bigvee_{k=0}^{n} \xi\left(-i(|l|+1)+z_{l} k, i(|r|+1)+z_{r} k\right)^{(i)} .
$$

Since now $z_{l} \leq 0, z_{r} \geq 0$ we obtain

$$
\bigvee_{k=0}^{n} \widehat{\tau}^{-p k} \widehat{T}^{-q k} \xi_{i}(-i, i) \preccurlyeq \xi\left(-i(|l|+1)+z_{l} n, i(|r|+1)+z_{r} n\right)^{(i)}, \quad n, i \geq 0
$$


and so proceeding as in the previous cases we get

$$
h_{\vec{v}}(\Phi) \leq\left(z_{r}-z_{l}\right) \log a .
$$

In order to prove the desired estimates in the general case we first extend them from the case $\vec{v} \in \mathbb{Z}^{2}$ to $\vec{v} \in \mathbb{Q}^{2}$ by applying (1) and then to the general case $\vec{v} \in \mathbb{R}^{2}$ by the continuity of the directional entropy.

In the proof of Theorem 1 we shall use the following

REMARK 1. If $T$ is an automorphism of a Lebesgue probability space $(X, \mathcal{B}, \mu)$ and $\alpha$ is a finite measurable partition of $X$ such that for some $N \in \mathbb{N}$ the partitions $\bigvee_{k=-n}^{0} T^{k} \alpha$ and $\bigvee_{k=N}^{N+n} T^{k} \alpha$ are independent for any $n \in \mathbb{N}$ then the partitions $\left(T^{t N} \alpha, t \in \mathbb{Z}\right)$ are independent.

We shall assume in what follows that the Borel measure considered, denoted by $\mu$, is uniform Bernoulli.

THEOREM 1. If the automaton rule $F_{[l, r]}$ is right permutative then

$$
h_{\vec{v}}(\Phi)=\left|z_{r}\right| \log a
$$

for all $\vec{v}=(x, y)$ with $0 \leq z_{l} \leq z_{r}$ or $z_{r} \leq z_{l} \leq 0$.

Proof. It suffices to show, using the Proposition, the inequality

$$
h_{\vec{v}}(\Phi) \geq\left|z_{r}\right| \log a .
$$

It is enough to consider the case $\vec{v} \in \mathbb{Z}^{2}$ and $0 \leq z_{l} \leq z_{r}$. One extends the above estimate to the general case as in the proof of the Proposition.

Let $\vec{v}=(p, q) \in \mathbb{Z}^{2}, 0 \leq z_{l}=p+q l \leq z_{r}=p+q r$ and let $i \in \mathbb{N}$. First we show that for $N=\left[2 i / z_{r}\right]+1$ the partitions

$$
\bigvee_{k=-n}^{0} \widehat{\tau}^{p k} \widehat{T}^{q k} \xi(-i, i)^{(0)} \text { and } \bigvee_{k=N}^{N+n} \widehat{\tau}^{p k} \widehat{T}^{q k} \xi(-i, i)^{(0)}
$$

are independent for any $n \in \mathbb{N}$. Since $0 \leq z_{l} \leq z_{r}$ we may proceed in the same way as in case A of the proof of the Proposition. We have

$$
\bigvee_{k=-n}^{0} \widehat{\tau}^{p k} \widehat{T}^{q k} \xi(-i, i)^{(0)} \preccurlyeq \bigvee_{k=0}^{n} \xi\left(-i+z_{l} k, i+z_{r} k\right)^{(0)} \preccurlyeq \xi\left(-i, i+z_{r} n\right)^{(0)}
$$

On the other hand,

$$
\begin{aligned}
\bigvee_{k=N}^{N+n} \widehat{\tau}^{p k} \widehat{T}^{q k} \xi(-i, i)^{(0)} & =\bigvee_{k=N}^{N+n} \widehat{T}^{q k} \xi(-i-p k, i-p k)^{(0)} \\
& =\bigvee_{k=N}^{N+n} \widehat{T}^{-q(N+n-k)} \xi(-i-p k, i-p k)^{(q N+q n)}
\end{aligned}
$$




$$
\begin{aligned}
& \preccurlyeq \bigvee_{k=N}^{N+n} \xi(-i-p k+l q(N+n-k), i-p k+r q(N+n-k))^{(q N+q n)} \\
& =\bigvee_{k=N}^{N+n} \xi\left(-i+l q(N+n)-z_{l} k, i+r q(N+n)-z_{r} k\right)^{(q N+q n)} \\
& \preccurlyeq \xi\left(-i+l q(N+n)-z_{l}(N+n), i+r q(N+n)-z_{r} N\right)^{(q N+q n)} \\
& =\xi(-i-p(N+n), i+r q n-p N)^{(q N+q n)} .
\end{aligned}
$$

Putting

$$
\begin{array}{lll}
i_{1}=-i-p(N+n), & j_{1}=i+r q n-p N, & m=q N+q n \\
i_{2}=-i, & j_{2}=i+z_{r} n, & m^{\prime}=0,
\end{array}
$$

we get

$$
\begin{aligned}
\left(m-m^{\prime}\right) r & =(q N+q n) r=z_{r} N-p N+q n r \\
& =z_{r}\left(\left[2 i / z_{r}\right]+1\right)-p N+q n r \\
& >2 i-p N+q n r=j_{1}-i_{2} .
\end{aligned}
$$

Therefore, by Lemma 2 the partitions

$$
\xi\left(-i, i+z_{r} n\right)^{(0)} \quad \text { and } \quad \xi(-i-p(N+n), i+r q n-p N)^{(q N+q n)}
$$

and hence the partitions

$$
\left.\bigvee_{k=-n}^{0} \widehat{\tau}^{p k} \widehat{T}^{q k} \xi(-i, i)^{(0)} \text { and } \bigvee_{k=N}^{N+n} \widehat{\tau}^{p k} \widehat{T}^{q k} \xi(-i, i)\right)^{(0)}
$$

are independent for any $n \in \mathbb{N}$. Applying Remark 1 we see that

$$
\left(\widehat{\tau}^{p N} \widehat{T}^{q N}\right)^{t} \xi(-i, i)^{(0)}, \quad t \in \mathbb{Z}
$$

are independent. Hence we get

$$
\begin{aligned}
h_{\vec{v}}(\Phi) & =h\left(\widehat{\tau}^{p} \widehat{T}^{q}\right)=\frac{1}{N} h\left(\widehat{\tau}^{p N} \widehat{T}^{q N}\right) \\
& \geq \frac{1}{N} h_{\widehat{\mu}}\left(\xi(-i, i)^{(0)}, \widehat{\tau}^{p N} \widehat{T}^{q N}\right)=\frac{1}{N} H_{\widehat{\mu}}\left(\xi(-i, i)^{(0)}\right) \\
& =\frac{1}{N} H_{\mu}(\xi(-i, i))=\frac{1}{N} \log a^{2 i+1}=(2 i+1)\left(\left[2 i / z_{r}\right]+1\right)^{-1} \log a
\end{aligned}
$$

for any $i \geq 0$. Taking the limit as $i \rightarrow \infty$ we obtain $h_{\vec{v}}(\Phi) \geq z_{r} \log a$.

REMARK 2. Using similar arguments one can show that if the automaton rule $F_{[l, r]}$ is left permutative then $h_{\vec{v}}(\Phi)=\left|z_{l}\right| \log a$ for all $\vec{v}=(x, y)$ with $z_{l} \leq z_{r} \leq 0$ or $0 \leq z_{r} \leq z_{l}$.

Now we shall prove the following 
THEOREM 2. If the automaton rule $F_{[l, r]}$ is bipermutative and $z_{l} \cdot z_{r} \leq 0$, then

$$
h_{\vec{v}}(\Phi)=\left|z_{r}-z_{l}\right| \log a .
$$

In order to show this equality we give two lemmas which are generalizations of results from [1]. Their proofs are slight modifications of the proofs of Lemmas 1 and 3 of [1].

Let $\alpha \in(0,1)$ be fixed and let $d_{\alpha}$ be the distance in $X$ defined by

$$
d_{\alpha}(x, y)= \begin{cases}\alpha^{L(x, y)}, & x \neq y \\ 0, & x=y\end{cases}
$$

where

$$
L(x, y)=\min \left\{|i| ; x_{i} \neq y_{i}\right\}, \quad x, y \in X .
$$

In the following, we use the notation $T_{F}$ instead of $T$.

Lemma 3. If $l<0<r, F=F_{[l, r]}$ is bipermutative and $\xi$ is the zero-time partition of $X$ then for every cylindric set $C \in \xi(l, r-1)$ we have

(i) $T_{F} C=X$,

(ii) $d_{\alpha}\left(T_{F} x, T_{F} y\right) \geq \alpha^{-\min (-l, r)} d_{\alpha}(x, y), x, y \in C$.

Proof. Let $u \in X$. We show that there exists a unique $x \in C$ with $T_{F} x=u$. Let

$$
C=C_{\bar{x}_{l}, \ldots, \bar{x}_{r-1}}=\left\{x \in X ; x_{l}=\bar{x}_{l}, \ldots, x_{r-1}=\bar{x}_{r-1}\right\} .
$$

The sequence $x$ is defined as follows. Let

$$
x_{l}=\bar{x}_{l}, \ldots, x_{r-1}=\bar{x}_{r-1} .
$$

Applying the right (left) permutativity of $F$ the reader can easily find by induction the coordinates $x_{k}, k \geq r(k \leq l-1)$, of $x$ in such a way that $T_{F} x=u, x \in C$, i.e. (i) is satisfied.

In order to show (ii) we take $x, y \in C$ with $x \neq y$. If $L(x, y)=L$ we have $d(x, y)=\alpha^{L}$ and either $x_{L} \neq y_{L}$ or $x_{-L} \neq y_{-L}$.

Let $x_{L} \neq y_{L}$. Since $x, y \in C$ we have $L \geq r$ and so by the definition of $L$,

$$
y_{L-r+l}=x_{L-r+l}, \ldots, y_{L-1}=x_{L-1} .
$$

Thus the right permutativity of $F$ implies

$$
\left(T_{F} x\right)_{L-r}=F\left(x_{L-r+l}, \ldots, x_{L}\right) \neq F\left(y_{L-r+l}, \ldots, y_{L}\right)=\left(T_{F} y\right)_{L-r} .
$$

Therefore

$$
d_{\alpha}\left(T_{F} x, T_{F} y\right) \geq \alpha^{L-r}=\alpha^{-r} d(x, y)
$$

and so the desired inequality is satisfied.

If $x_{-L} \neq y_{-L}$ then similarly we have $-L<l$, and

$$
x_{-L+1}=y_{-L+1}, \ldots, x_{-l-L+r}=y_{-l-L+r} .
$$


The left permutativity of $F$ gives

$$
\left(T_{F} x\right)_{-l-L} \neq\left(T_{F} y\right)_{-l-L} .
$$

Hence

$$
d_{\alpha}\left(T_{F} x, T_{F} y\right) \geq \alpha^{L-|l|}=\alpha^{-|l|} d_{\alpha}(x, y)
$$

and thus we obtain the needed inequality again.

Let $\alpha$ be a finite measurable partition of $X$. Points $x, y \in X$ are said to be $\alpha$-separated if they belong to different atoms of $\alpha$.

It is easy to see that for partitions $\alpha, \beta \in X$ we have $\alpha \preccurlyeq \beta$ iff any $\alpha$-separated points $x, y \in X$ are also $\beta$-separated.

Lemma 4. If $l<0<r$ and $F=F_{[l, r]}$ is bipermutative, then

$$
h\left(T_{F}\right) \geq(r-l) \log a .
$$

Proof. First we show that

$$
\bigvee_{k=0}^{n-1} T_{F}^{-k} \xi(l, r) \succcurlyeq \xi(n l, n r)
$$

for every $n \geq 1$ where $\xi$ is the zero-time partition of $X$.

The above property is trivially satisfied for $n=1$. Suppose it is true for some $n \geq 1$ and let $x, y \in X$ be $\xi((n+1) l,(n+1) r)$-separated.

If $x, y$ are $\xi(n l, n r)$-separated then they are also $\bigvee_{k=0}^{n} T_{F}^{-k} \xi(l, r)$-separated by the induction assumption. So suppose $x, y$ are not $\xi(n l, n r)$ separated. It follows from Lemma 3 that

$$
\begin{aligned}
d\left(T_{F} x, T_{F} y\right) & \geq \alpha^{-\min (-l, r)} d(x, y) \geq \alpha^{-\min (-l, r)} \cdot \alpha^{(n+1) \min (-l, r)} \\
& =\alpha^{n \min (-l, r)},
\end{aligned}
$$

i.e. $T_{F} x, T_{F} y$ are $\xi(n l, n r)$-separated. Therefore $x, y$ are $\bigvee_{k=0}^{n} T_{F}^{-k} \xi(l, r)$ separated, i.e. (3) is valid for all $n$.

Applying (3) we have

$$
\begin{aligned}
h\left(T_{F}\right) & \geq \lim _{n \rightarrow \infty} \frac{1}{n} H_{\mu}\left(\bigvee_{k=0}^{n-1} T_{F}^{-k} \xi(l, r)\right) \geq \lim _{n \rightarrow \infty} \frac{1}{n} H_{\mu}(\xi(n l, n r)) \\
& =(r-l) \log a .
\end{aligned}
$$

Corollary. If $-r<k<-l$ then

$$
h\left(\tau^{k} T_{F}\right) \geq(r-l) \log a .
$$

Proof. It follows from the inequalities $l+k<0<r+k$ and the equality

$$
\tau^{k} T_{F[l, r]}=T_{F[l+k, r+k]}
$$

that $\tau^{k} T_{F[l, r]}$ is a bipermutative automaton map which satisfies the assumption of Lemma 4 . Hence we obtain at once the desired inequality. 
Proof of Theorem 2. It is enough to consider the case $\vec{v}=(p, q) \in \mathbb{Z}^{2}$ where $p+l q<0<p+r q$. It follows from the Corollary to Lemma 4 and the fact that $\tau$ is an automorphism commuting with $T_{F}$ that

$$
\begin{aligned}
h_{\vec{v}}(\Phi) & =h_{\widehat{\mu}}\left(\widehat{\tau}^{p} \widehat{T}_{F[l, r]}^{q}\right)=h_{\mu}\left(\tau^{p} T_{F[l, r]}^{q}\right)=h_{\mu}\left(\tau^{p} T_{F[q l, q r]}\right) \\
\geq q(r-l) \log a & =\left(z_{r}-z_{l}\right) \log a .
\end{aligned}
$$

\section{References}

[1] V. S. Afraimovich and M. A. Shereshevsky, Bipermutative cellular automata are topologically conjugate to the one-sided Bernoulli shift, Random Comput. Dynam. 1 (1992-93), 91-98.

[2] M. Boyle and D. Lind, Expansive subdynamics, Trans. Amer. Math. Soc. 349 (1997), 55-102.

[3] E. M. Coven and M. Paul, Endomorphisms of irreducible shifts of finite type, Math. Systems Theory 8 (1974), 165-177.

[4] G. A. Hedlund, Endomorphisms and automorphisms of the shift dynamical system, ibid. 3 (1969), 320-375.

[5] J. Milnor, Directional entropies of cellular automaton-maps, in: Disordered Systems and Biological Organization, NATO Adv. Sci. Inst. Ser. F 20, Springer, 1986, 113115 .

[6] - On the entropy geometry of cellular automata, Complex Systems 2 (1988), 357386.

[7] K. K. Park, On directional entropy functions, Israel J. Math. 113 (1999), 243-267.

[8] V. A. Rokhlin, Lectures on the theory of entropy of transformations with invariant measure, Uspekhi Mat. Nauk 22 (1967), no. 5, 3-56 (in Russian).

[9] M. A. Shereshevsky, Ergodic properties of certain surjective cellular automata, Monatsh. Math. 114 (1992), 305-316.

Laboratoire de Physique Théorique de la Matière Condensée

Université Paris VII

Tour 24, 5ème étage, porte 506

B.P. 7020

Place Jussieu

75251 Paris Cedex, France

E-mail: courbage@ccr.jussieu.fr
Faculty of Mathematics and Computer Science

Nicholas Copernicus University Chopina 12/18

87-100 Toruń, Poland E-mail: bkam@mat.uni.torun.pl

Received October 8, 2001

Revised version February 25, 2002 